\title{
Progress in Reducing Aerodynamic Drag for Higher Efficiency of Heavy Duty Trucks (Class 7-8)
}

\author{
Rose McCallen, Fred Browand \\ Mustapha Hammache, Anthony Leonard \\ Mark Brady, Kambiz Salari \\ Walter Rutledge, James Ross \\ Bruce Storms, J.T. Heineck
}

This paper was prepared for submittal to the

1999 Society of Automotive Engineers

Government/Industry Meeting

Washington, DC

April 26-28, 1999

April 1999

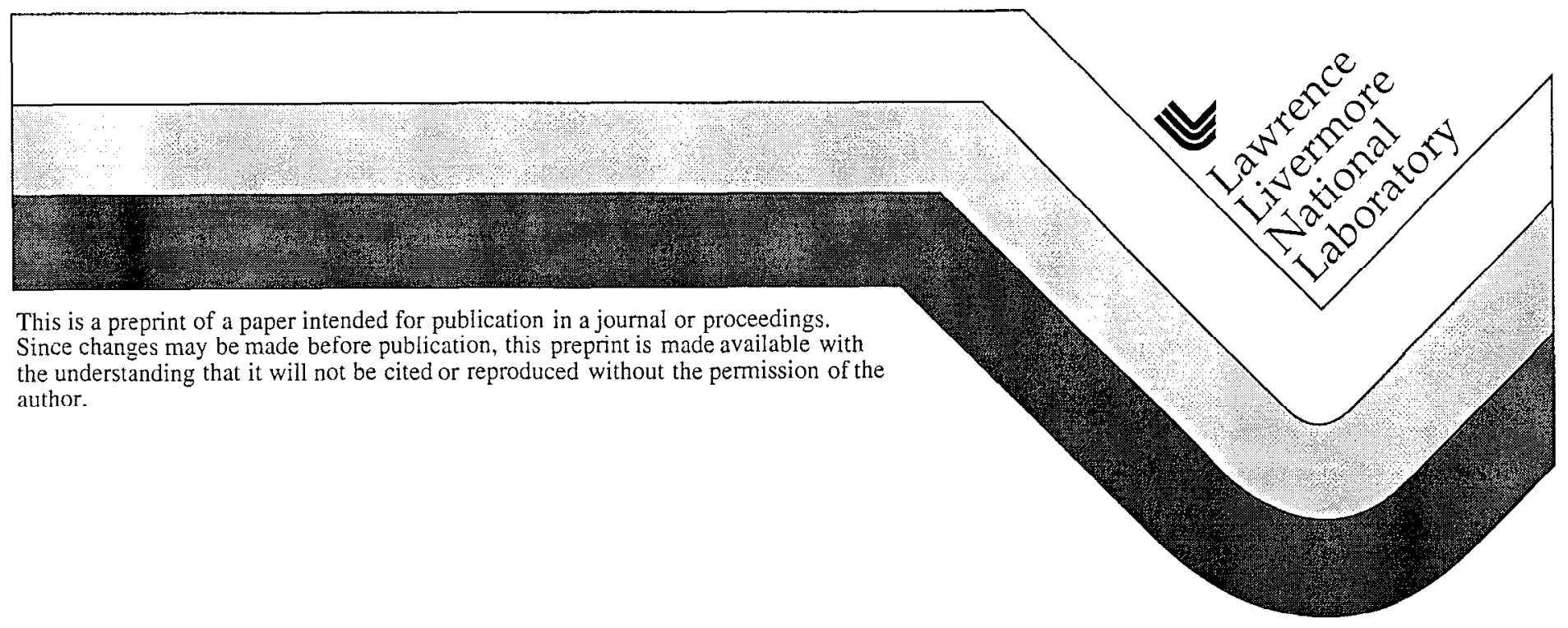




\section{DISCLAIMER}

This document was prepared as an account of work sponsored by an agency of the United States Government. Neither the United States Government nor the University of California nor any of their employees, makes any warranty, express or implied, or assumes any legal liability or responsibility for the accuracy, completeness, or usefulness of any information, apparatus, product, or process

disclosed, or represents that its use would not infringe privately owned rights. Reference herein to any specific commercial product, process, or service by trade name, trademark, manufacturer, or otherwise, does not necessarily constitute or imply its endorsement, recommendation, or favoring by the United States Government or the University of California. The views and opinions of authors expressed herein do not necessarily state or reflect those of the United States Government or the University of California, and shall not be used for advertising or product endorsement purposes. 


\title{
PROGRESS IN REDUCING AERODYNAMIC DRAG FOR HIGHER EFFICIENCY OF HEAVY DUTY TRUCKS (CLASS 7-8)
}

\author{
Rose McCallen \\ Lawrence Livermore National Laboratory, Livermore, CA \\ Fred Browand and Mustapha Hammache \\ University of Southern California, Los Angeles, CA \\ Anthony Leonard and Mark Brady \\ California Institute of Technology, Pasadena, CA \\ Kambiz Salari and Walter Rutledge \\ Sandia National Laboratories, Albuquerque, NM \\ James Ross, Bruce Storms ${ }^{1}$, and J.T. Heineck \\ NASA Ames Research Center, Moffett Field, CA
}

This paper describes research and development for reducing the aerodynamic drag of heavy vehicles by demonstrating new approaches for the numerical simulation and analysis of aerodynamic flow. In addition, greater use of newly developed computational tools holds promise for reducing the number of prototype tests, for cutting manufacturing costs, and for reducing overall time to market.

Experimental verification and validation of new computational fluid dynamics methods are also an important part of this approach. Experiments on a model of an integrated tractor-trailer are underway at NASA Ames Research Center and the University of Southern California. Companion computer simulations are being performed by Sandia National Laboratories, Lawrence Livermore National Laboratory, and California Institute of Technology using state-of-the-art techniques, with the intention of implementing more complex methods in the future.

1. On contract to NASA Ames from Aerospace Computing, Inc. 


\section{Introduction and Background}

Reduced fuel consumption for heavy vehicles can be achieved by altering truck shapes to decrease the aerodynamic resistance (drag). It is conceivable that present day truck drag coefficients can be reduced by as much as $50 \%$, which represents a fuel savings of three billion gallons of diesel fuel per year of the roughly 15 billion gallons now consumed for truck highway travel at speeds of 60 miles per hour.

The aerodynamic design of heavy trucks is presently based upon estimations of performance derived from wind tunnel testing. No better methods have been available traditionally, and the designer/aerodynamicists are to be commended for achieving significant design improvements over the past several decades on the basis of limited quantitative information. Computer simulation of acrodynamic flow is a new possibility, but the truck manufacturers have not yet integrated state-of-the-art computational simulations into advanced design approaches to predict performance of optimized aerodynamic vehicles. This is due partly because currently available methods are not reliable in their predictions for complex tractor-trailer flows.

Figure 1 contains the estimated horsepower associated with aerodynamic drag in comparison to the power required to overcome rolling resistance and to supply needed auxiliary power, plotted as a function of speed.The truck in question is a modern Class 8 tractortrailer possessing a wind-averaged drag coefficient of $C_{D}=0.60$, and weighing 80,000 pounds. At 70 miles per hour, overcoming aerodynamic drag represents about $65 \%$ of the total energy expenditure for a typical heavy truck vehicle.

Figure 2 illustrates the overall economic benefit associated with reductions in aerodynamic drag as a function of vehicle speed. The ordinate on the left presents calculations of fuel consumption in gallons per mile traveled for a typical Class 8 tractor-trailer powered by a modern, turbocharged diesel engine operating at a fixed specific fuel consumption, $\mathrm{bsfc}=0.34 \# / \mathrm{HP}$-hr. Five estimates of fuel consumption are shown, corresponding to five values of wind-averaged drag coefficient between $C_{D}=0.7$ and 0.3 . To the right are plotted the total yearly fuel expenditures expressed in billions of gallons based upon the estimate of 60 billion highway miles traveled (per year) in the year 2012 by Class 8 trucks. The 60 billion highway miles is predicted by applying a 30\% growth factor to the FHWA annual vehicle-travel estimates for 1992 [1]. Reducing the drag coefficient from 0.6 to 0.3 for a typical Class 8 tractor-trailer would result in a total yearly savings of 3 billion gallons of diesel fuel for travel at a present day speed of 60 miles per hour. The mileage improvement is from 6.1 miles per gallon to 8.7 miles per gallon - a $43 \%$ savings.

\section{Experiments}

We present here an overview of the current experimental approach and several preliminary results provided for the integrated tractor-trailer benchmark geometry termed the Sandia Model [2]. The purpose of the tests are for validation of the computational fluid dynamics (CFD) models, and for further insight into truck flow phenomena. 


\section{Baseline Model in NASA Ames 7-ft. x 10-ft. Wind Tunnel}

NASA Ames' Experimental Physics Branch have recently performed experiments on the Sandia Model (a 1/8 scale model) in the 7-ft. x 10-ft. wind tunnel (see Figure 3). In addition to drag and discrete and unsteady pressure measurements, an entire suite of new and innovative measurement techniques were used. The three-dimensional (3D) unsteady wake was captured using particle image velocimetry (PIV). PIV is an imaging technique whose data product is usually an array of two-component velocity vectors on a given plane. PIV has become the flow field velocity measurement technique of choice for validating CFD code. A recent development in PIV is to derive the third component of velocity by using stereo imaging. This is the world's first 3D PIV system being used in a production wind tunnel in the world.

The PIV measurements were taken in the model wake, providing the three components of velocity in the plane of a laser sheet. PIV data was taken for Reynolds number $(\mathrm{Re})$ of 0.5 million and 2 million based on the trailer width and upstream velocity. In all, more than fifty data sets were collected. Examples of the PIV results are shown in Figures 4 and 5.

State-of-the-art oil film interferometry techniques (OFI) for measuring skin friction (Figure 6) and pressure sensitive paint (PSP) measurements (Figure 7) were also provided. The OFI technique can supply quantitative time-averaged skin friction measurements on the body and in the body wake. The OFI results shown in Figure 6 indicate a vortex on the top of the model at the 10-degree yaw condition. The PSP measurements provide timeaveraged pressures on the body.

Skin friction measurements on the model body were also provided by Tao Systems' hotfilm sensors which can detect flow separation, reattachment, and transition. A total of 60 sensors were used for the hot-film measurements (Figure 8).

Continuum Dynamics, Inc. has also provided boattail plates made to fit the Sandia Model (Figure 9). Tests conducted with and without the boattail plates show a 20\% reduction in drag when the plates are installed, as presented in Figure 10. (A 10\% reduction had previously been measured on a full-size truck of different design at similar speeds. The drag reduction is less for the full-scale case due to the more realistic truck geometry.)

\section{Baseline Model in USC Wind Tunnel}

In preparation for PIV measurements at USC, a 1:14 scale model of the Sandia Truck has been fabricated and instrumented to measure drag, side force, and yawing moment. The model has a separately instrumented tractor and trailer with the capability for varying the gap between the two. Preliminary measurements of drag as a function of tractor-trailer gap have been completed, and illustrated in Figure 11. The drag coefficients shown for the tractor (or cab) and the trailer are plotted separately, and normalized by their respective values at a typical gap - the value of 0.5 on the abscissa. (The normalizing length for the gap is the square root of the frontal area.) At large gaps, the trailer drag dominates, while at shorter gaps the cab drag is slightly greater than trailer drag. Interestingly, the drag curves cross again at very short gaps. (These results were provided by student researchers 
Glen Landreth and Patricia Wall.)

\section{Computations}

\section{Reynolds-Averaged Navier Stokes Modeling}

Reynolds-averaged Navier Stokes (RANS) computations are being performed by Sandia National Laboratories (SNL). Figure 12 shows a flow field simulation about the Sandia Model at Re of 1.6 million. The presented results involve the modeling of an experiment performed on the Sandia Model in the Texas A\&M 7-ft. x 10-ft. wind tunnel [2]. These RANS simulations include part of the converging section, test section, and part of the expansion region of the tunnel. The tunnel walls are treated as slip boundaries (no penetration). The computational meshes for the RANS simulations range from a coarse mesh of 0.5 million nodes, a medium mesh of 4 million nodes, and a fine mesh of 32 million nodes at 0 and 10 degree yaws. For these calculations an implicit finite-volume compressible flow solver with a one-equation Spalart-Allmaras turbulence model was used. The steady solutions were obtained on a massively parallel machine using 107 and 246 processors for the coarse and medium mesh, respectively. The fine mesh calculation is underway and it uses 1414 processors. Future plans are to use these solutions as the initial conditions for a time-accurate RANS calculations.

\section{Large-Eddy Simulation}

The large-eddy simulation (LES) approach is being used by Lawrence Livermore National Laboratory (LLNL). This advanced modeling approach has the potential to achieve more accurate simulations with minimum empiricism and thus, reduce experimentation. The flow around a tractor/trailer is time dependent, three-dimensional with a wide range of scales (i.e., the largest scale is on the order of the truck length and the small scales are smaller than the diameter of a grab handle). LLNL is utilizing an established finite element method. A preliminary mesh and calculations that demonstrate the benefits of the unstructured grid option and the ability of LES to capture the unsteady wake flow are presented in Figure 13.

An LES approach with vortex methods is being used by California Institute of Technology (Caltech). It is emphasized that this is truly a gridless method (except for the $2 \mathrm{D}$ grid on the vehicle surface). Gridless methods appear to be of particular interest to industry, because of the excess amount of time that is usually spent on mesh generation compared to the simulation run time. In addition, with vortex methods, computations are only performed where nonzero vorticity is present (e.g., near body and in wake) thus, reducing computational effort. In addition, there are other developments that reduce the effective operations from an order of $\mathrm{N}^{2}$ to order $\mathrm{N}$, where $\mathrm{N}$ is the number of computational elements (i.e., vortex packets) which move with the fluid.

\section{Summary, Conclusions, and Future Plans}


Experiments on a baseline case of an integrated tractor/trailer have been performed. In addition to drag and discrete and unsteady pressure measurements, an entire suite of new and innovative measurement techniques were used, including use of the world's first 3D PIV system in a production wind tunnel. The purpose of the tests are for validation of the CFD models and for further insight into truck flow phenomena.

Advanced computational models that use an LES approach are being developed, in addition to the use of state-of-the-art RANS modeling. This advanced LES modeling approach is being considered to achieve accurate simulations with minimum empiricism and thus, reduce experimentation and increase the understanding of contributory causes for drag of heavy trucks.

\section{Acknowledgment}

This project is supported by the Department of Energy, Office of Transportation Technology, Office of Heavy Vehicle Technology.

This work was performed under the auspices of the U.S. Department of Energy by Lawrence Livermore National Laboratory under Contract W-7405-Eng-48.

\section{References}

1. Highway Statistics 1992, p 207, US Government Printing Office, SSOP, Washington DC 20402-9328.

2. Croll, R. H., Gutierrez, W. T., Hassan, B., Suazo, J. E., and Riggins, A. J., "Experimental Investigation of the Ground Transportation Systems (GTS) Project for Heavy Vehicle Drag Reduction," SAE paper 960907, 1996. 


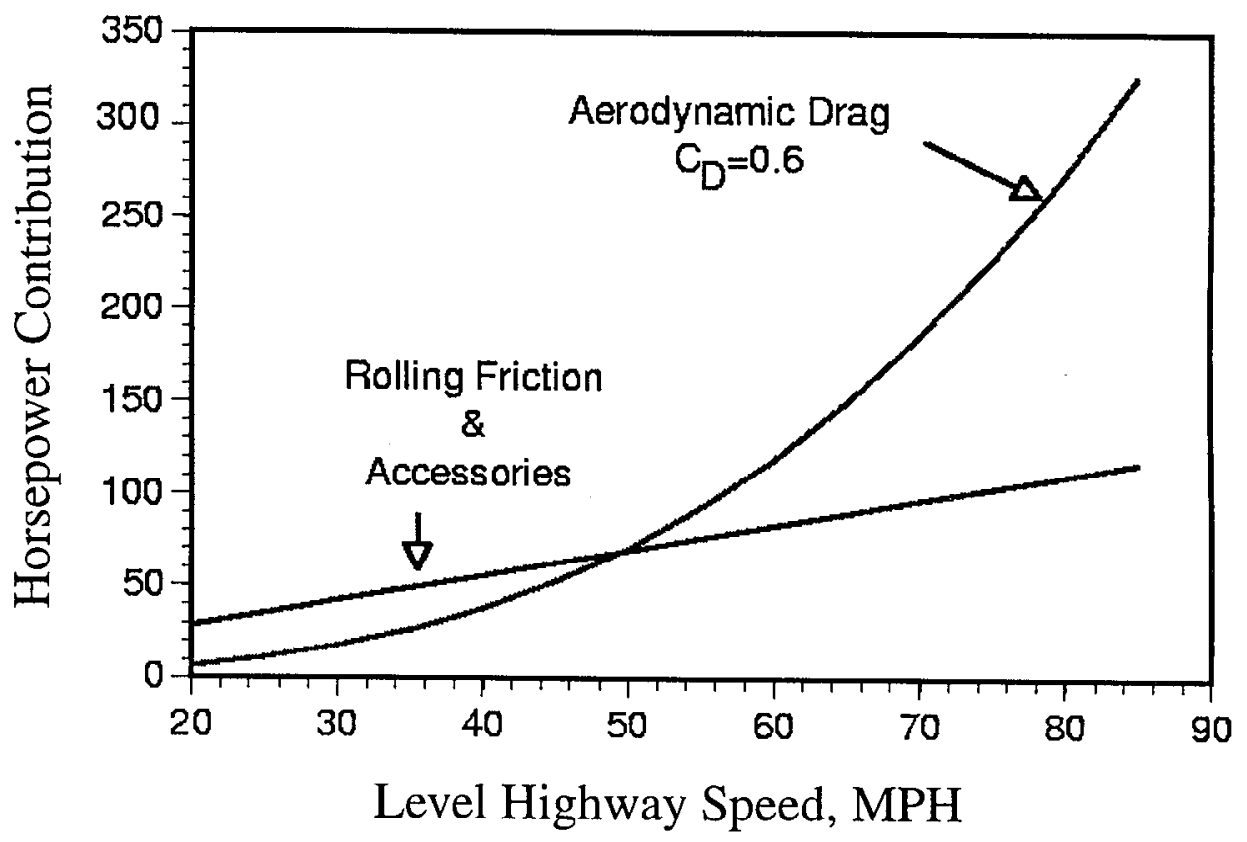

FIGURE 1. Horsepower required to overcome aerodynamic drag and rolling friction/accessories as a function of travel speed for a typical Class 8 tractor-trailer.

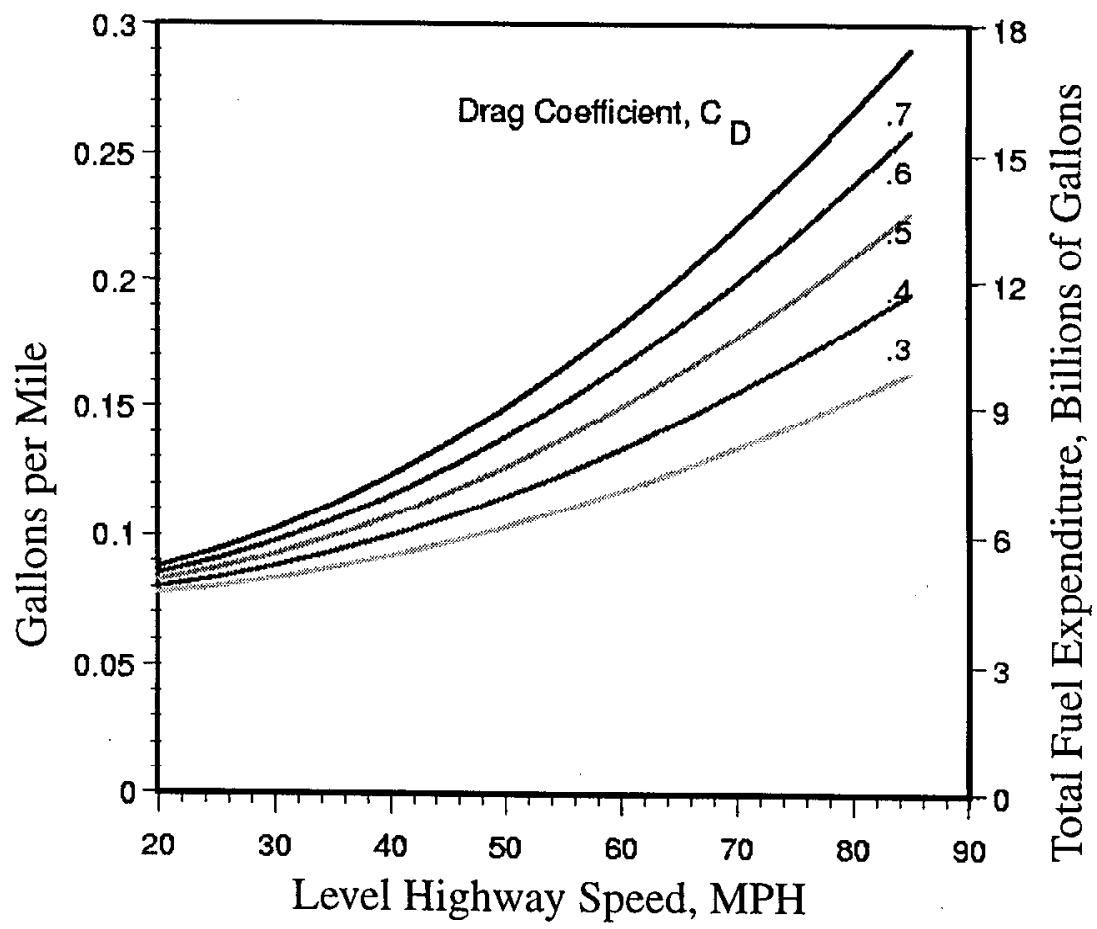

FIGURE 2. Fuel expenditures for a typical Class 8 tractor-trailer as a function of travel speed and drag coefficient. 


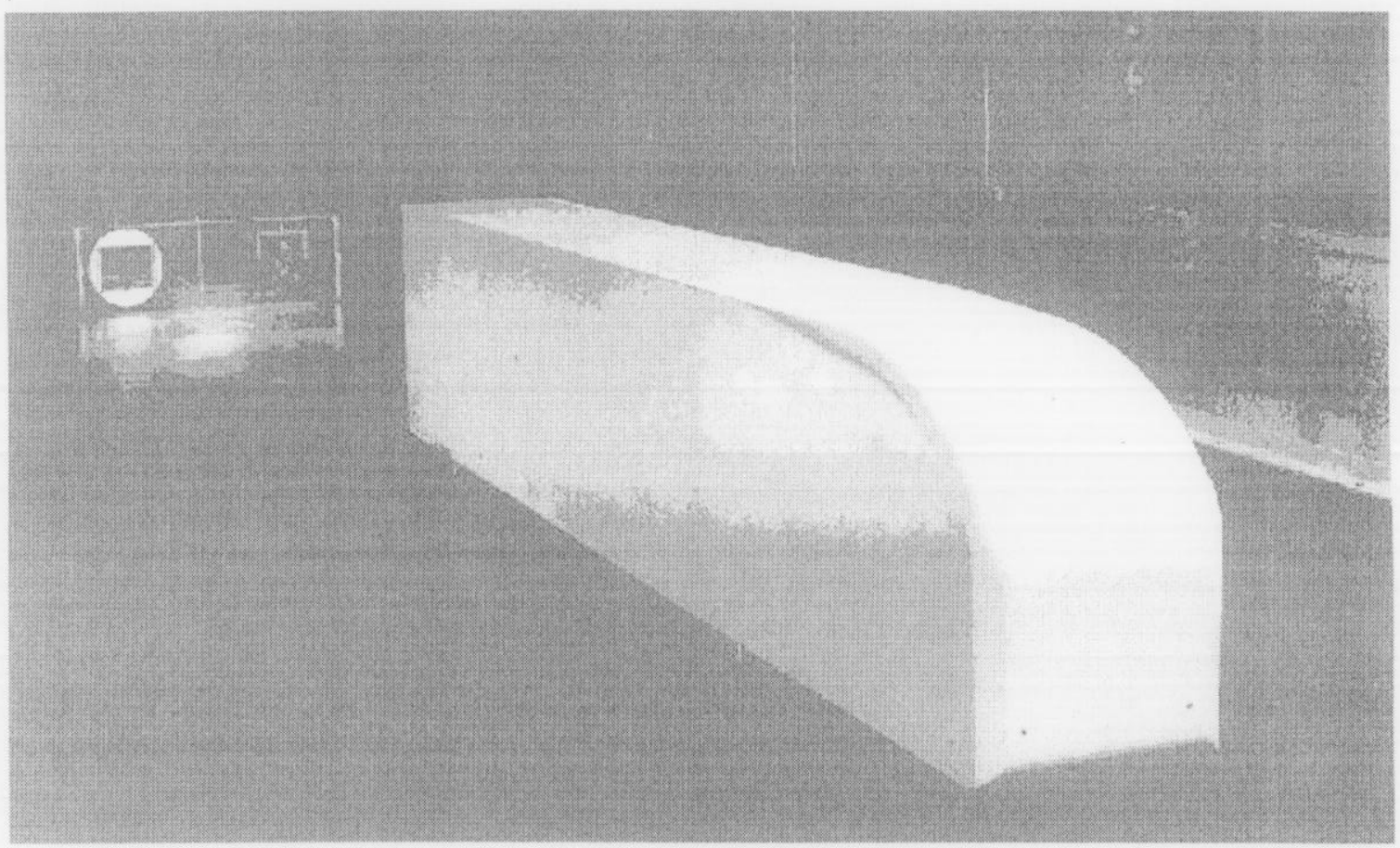

FIGURE 3. Sandia Model in NASA Ames 7-ft. x 10-ft. wind tunnel.

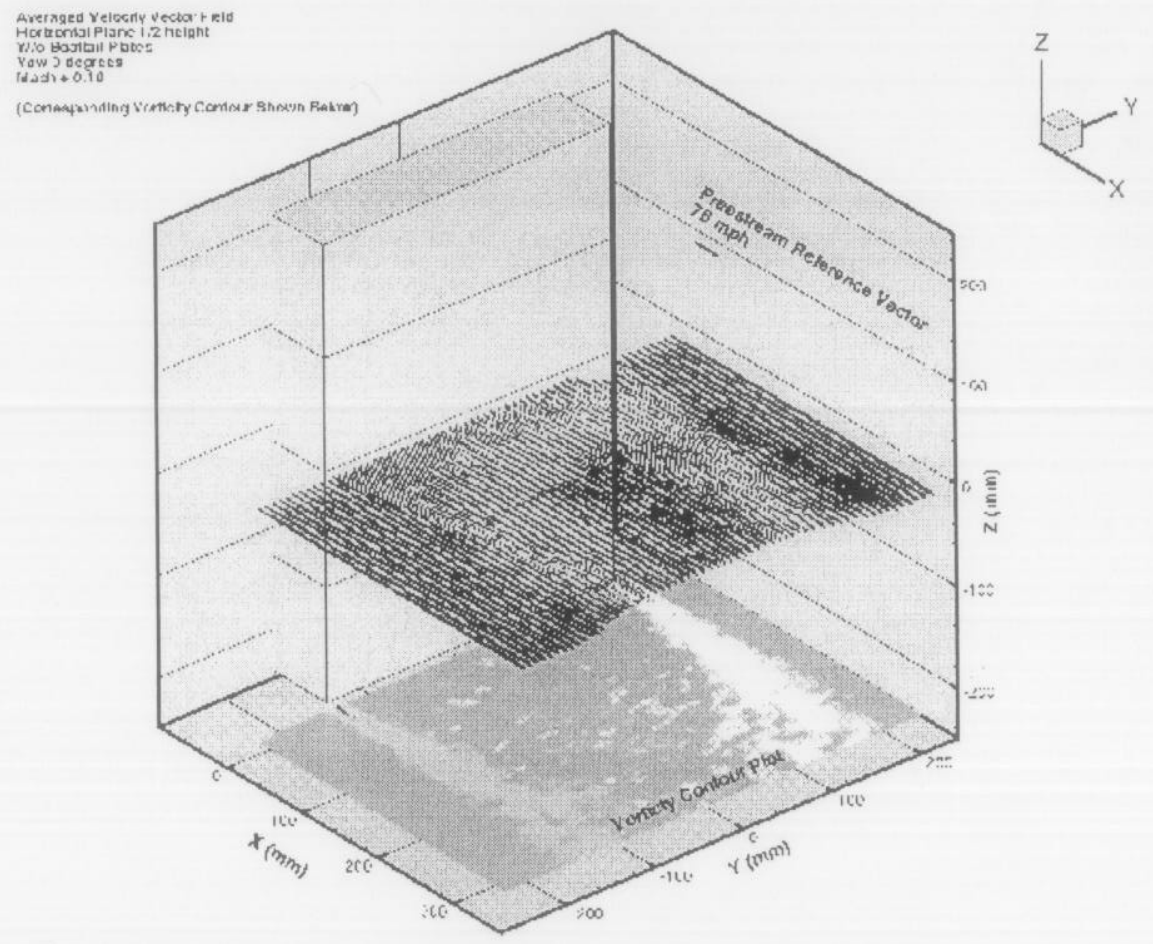

FIGURE 4. Time-averaged 3D vector velocity field and vorticity map for zero-degree yaw, baseline model case at Mach 0.1 . The laser plane horizontal at the half-height position. 


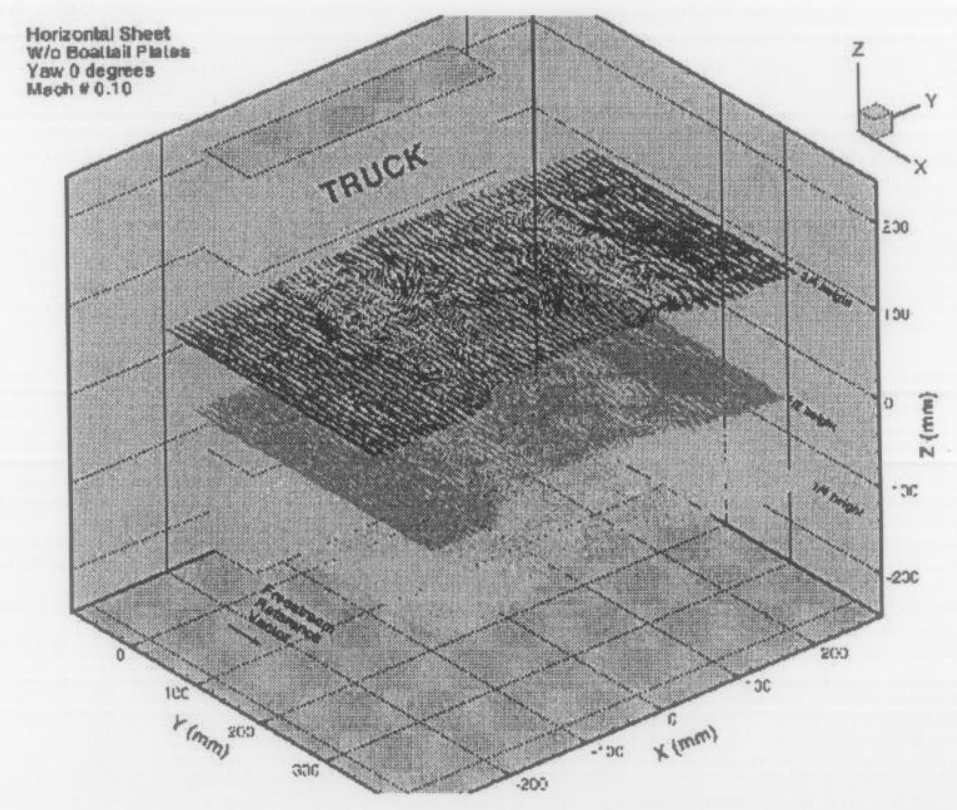

FIGURE 5. Three planes of instantaneous vector velocity fields for the zero-degree yaw, baseline model at Mach 0.1.

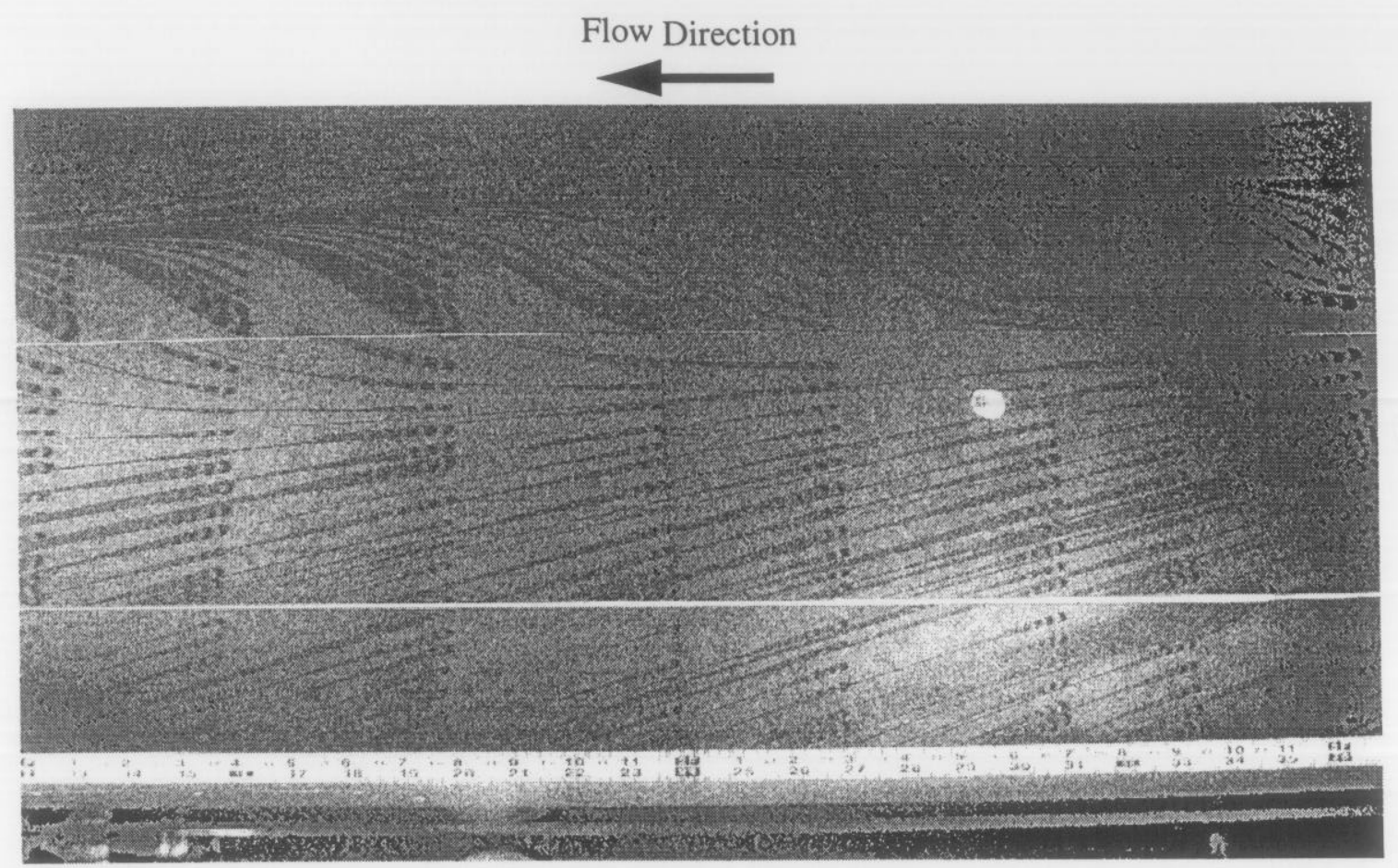

FIGURE 6. Oil film image for top view of trailer in NASA Ames' 7-ft. $x 10$-ft. wind tunnel at 10 degree yaw and Mach 0.27. The skin friction is proportional to the fringe spacing. 
Flow

Direction

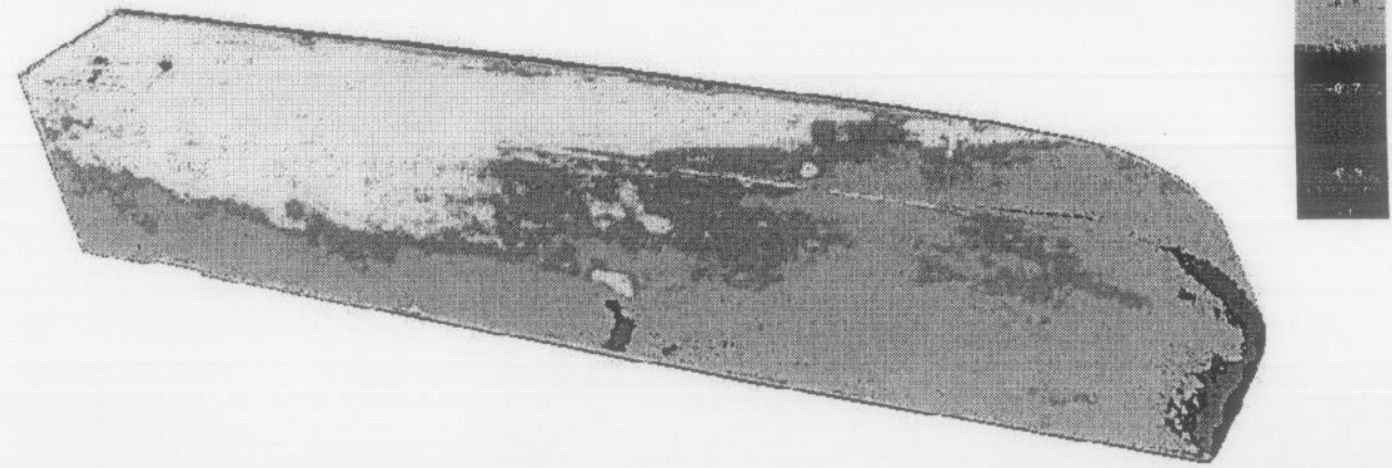

FIGURE 7. Pressure sensitive paint results on Sandia model in NASA Ames' 7-ft. x 10-ft. wind tunnel at 10 degree yaw and Mach 0.27 .

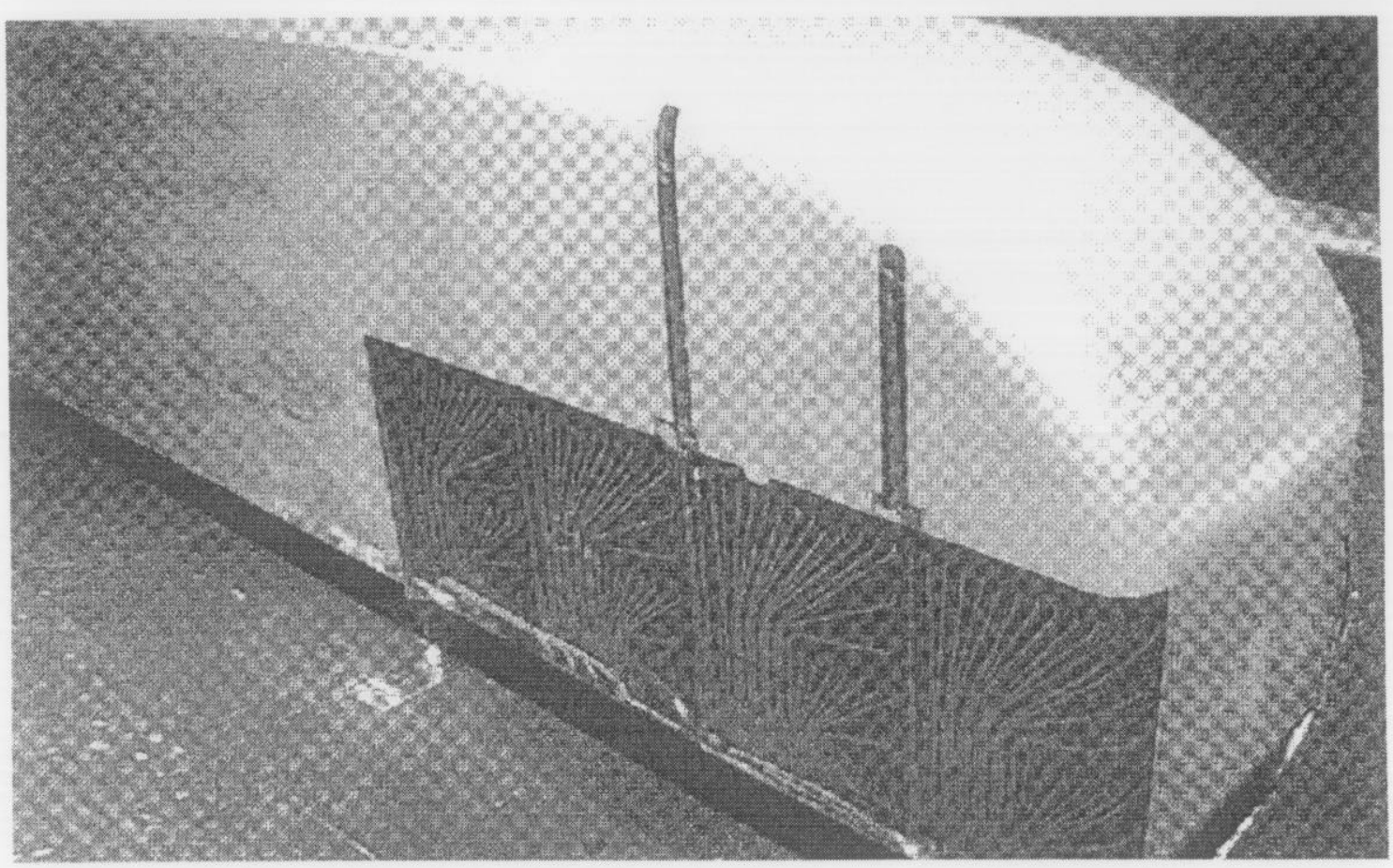

FIGURE 8. Hot film sensors on Sandia model in NASA Ames' 7-ft. x 10-ft. wind tunnel. 


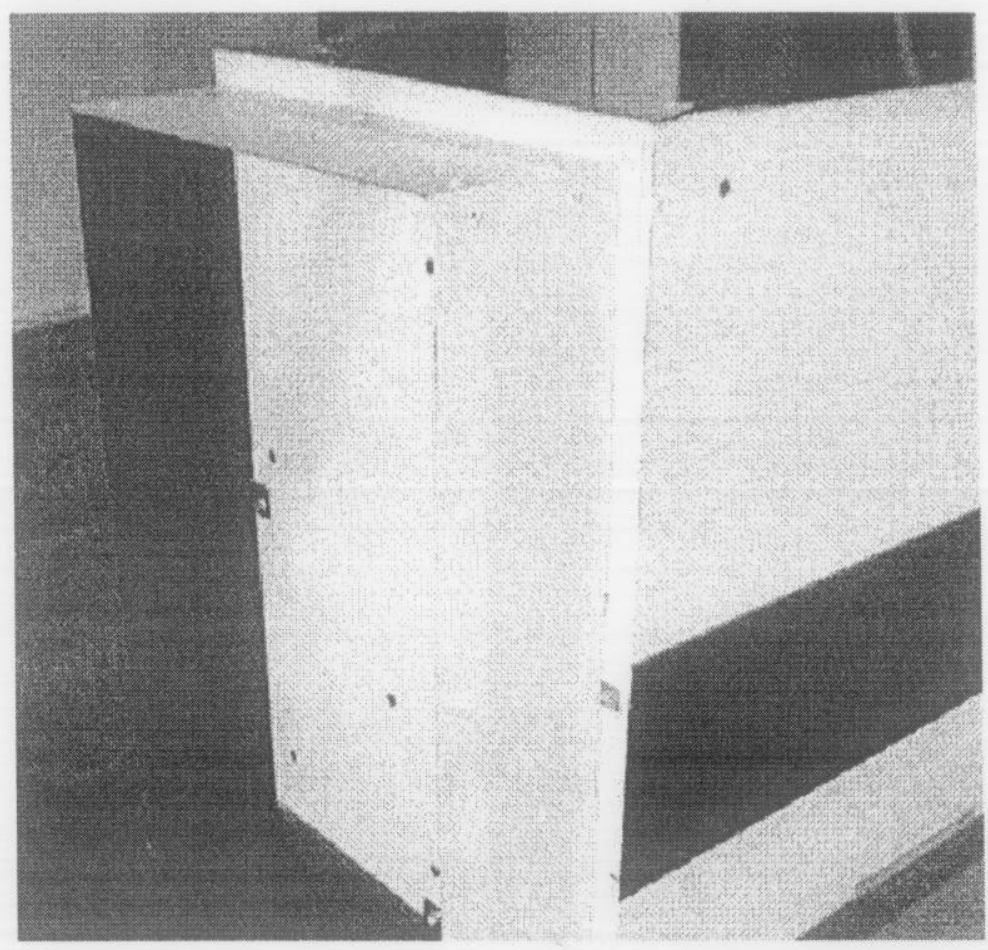

FIGURE 9. Continuum Dynamics, Inc. boattail plates installed on Sandia model.

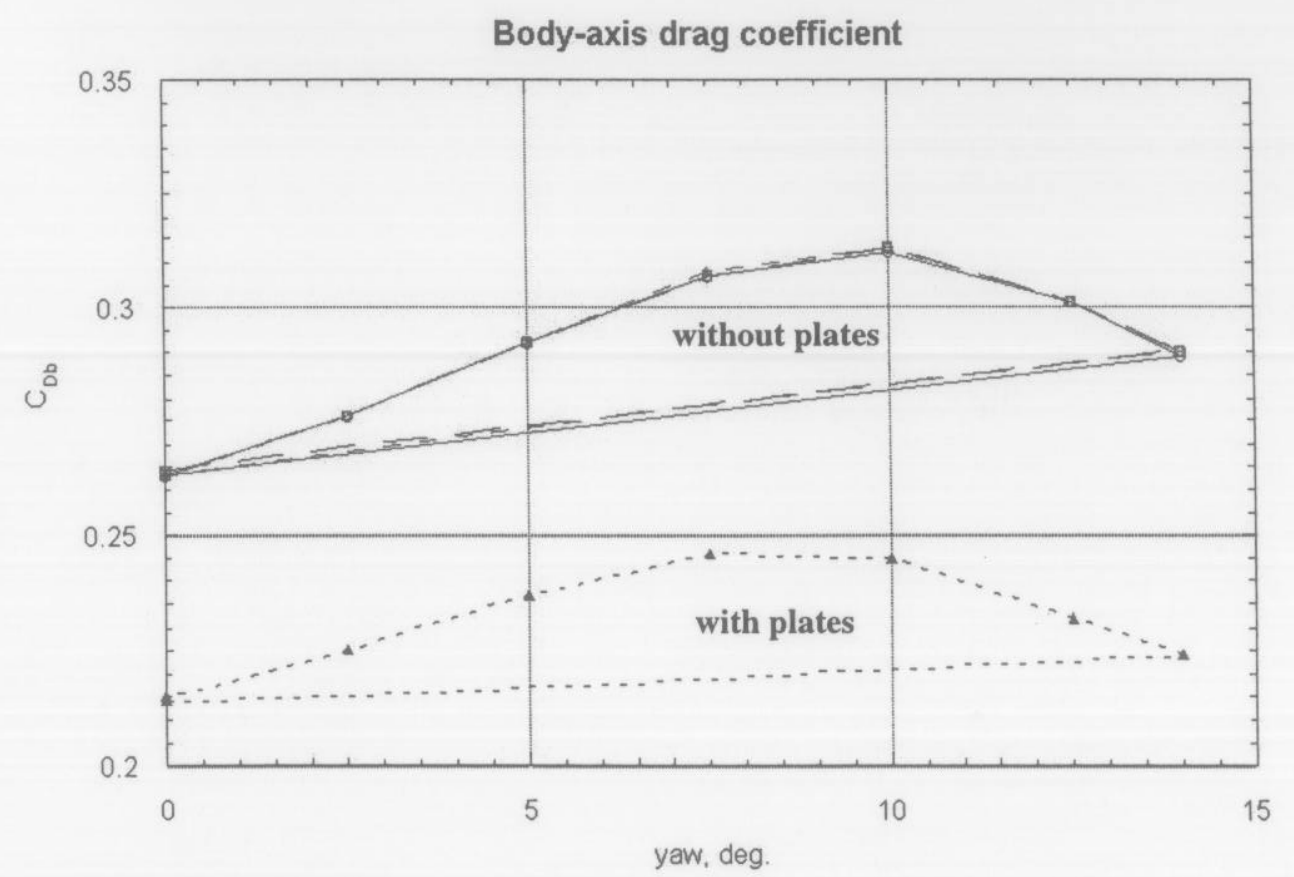

FIGURE 10. Effect of boattail plates on drag. 

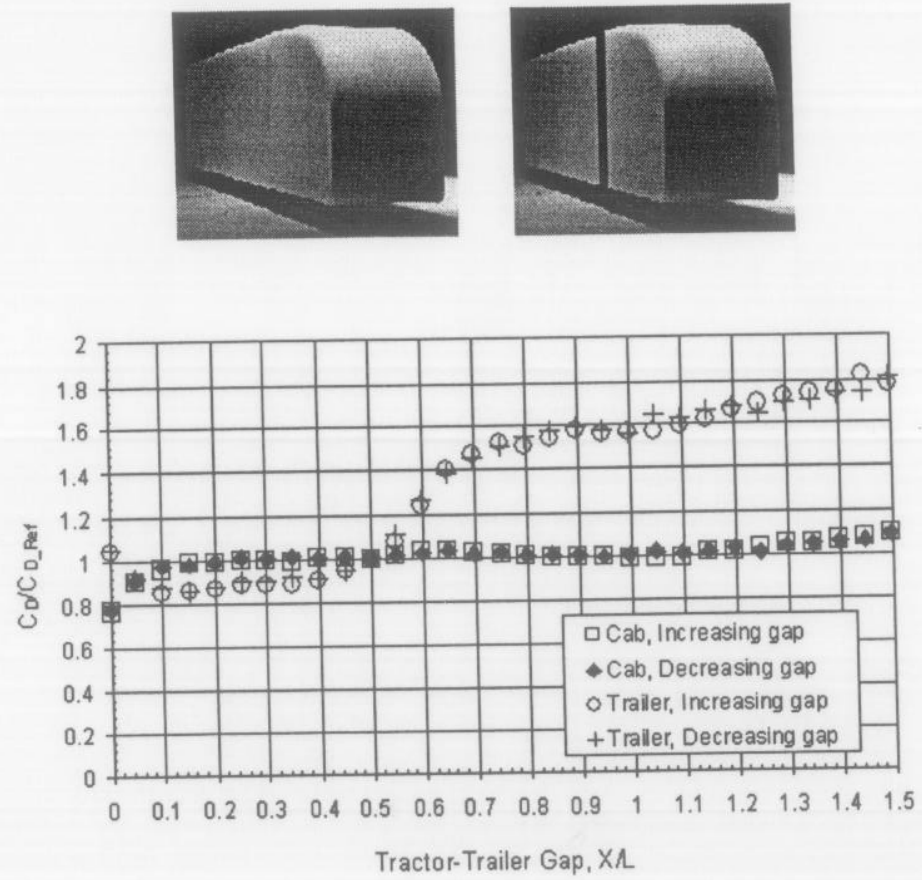

FIGURE 11. Drag coefficient ratios for tractor and trailer as a function of normalized gap length.

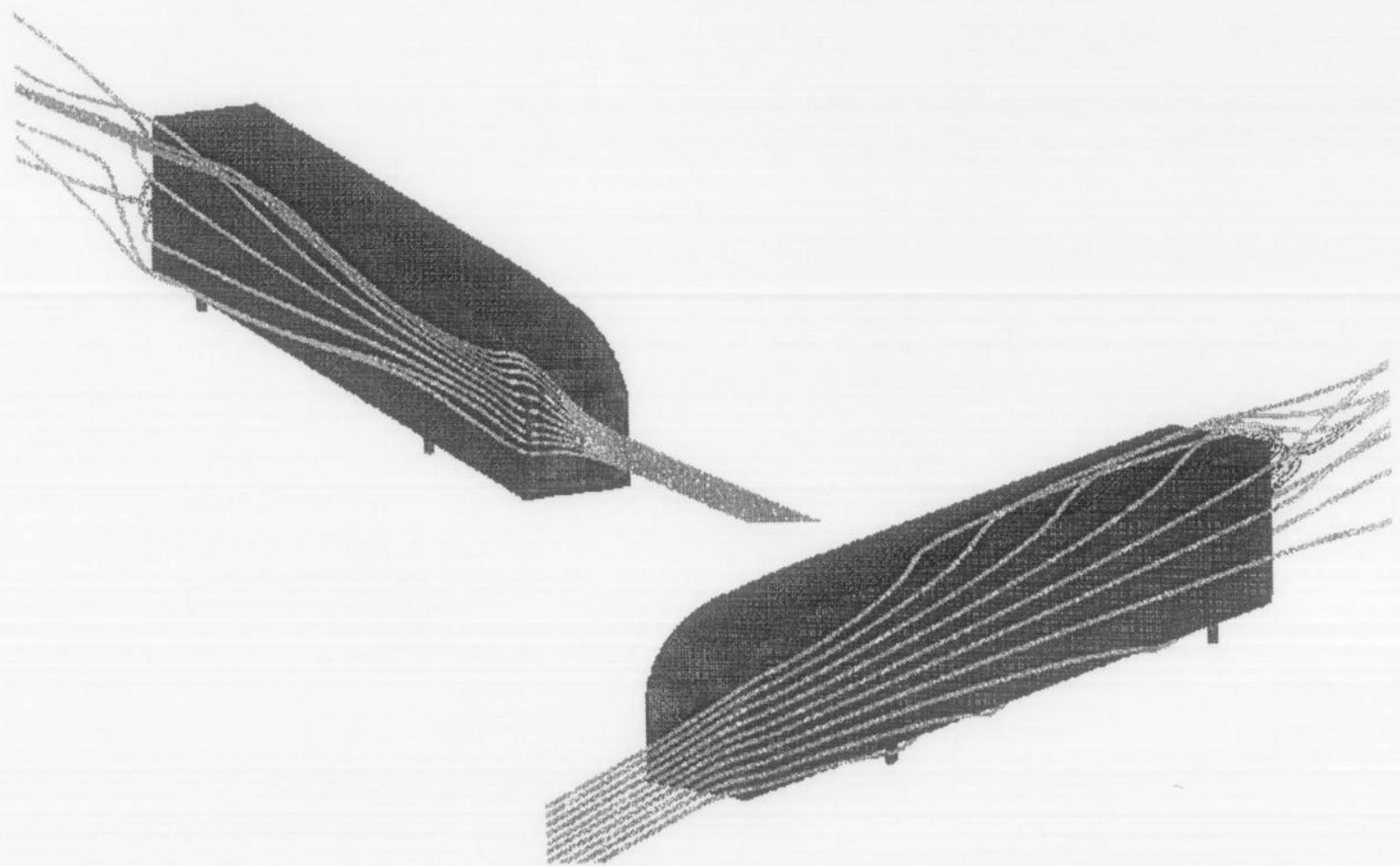

FIGURE 12. Reynolds-averaged Navier Stokes calculations of Sandia model for $\mathrm{Re}=1.6$ million. 


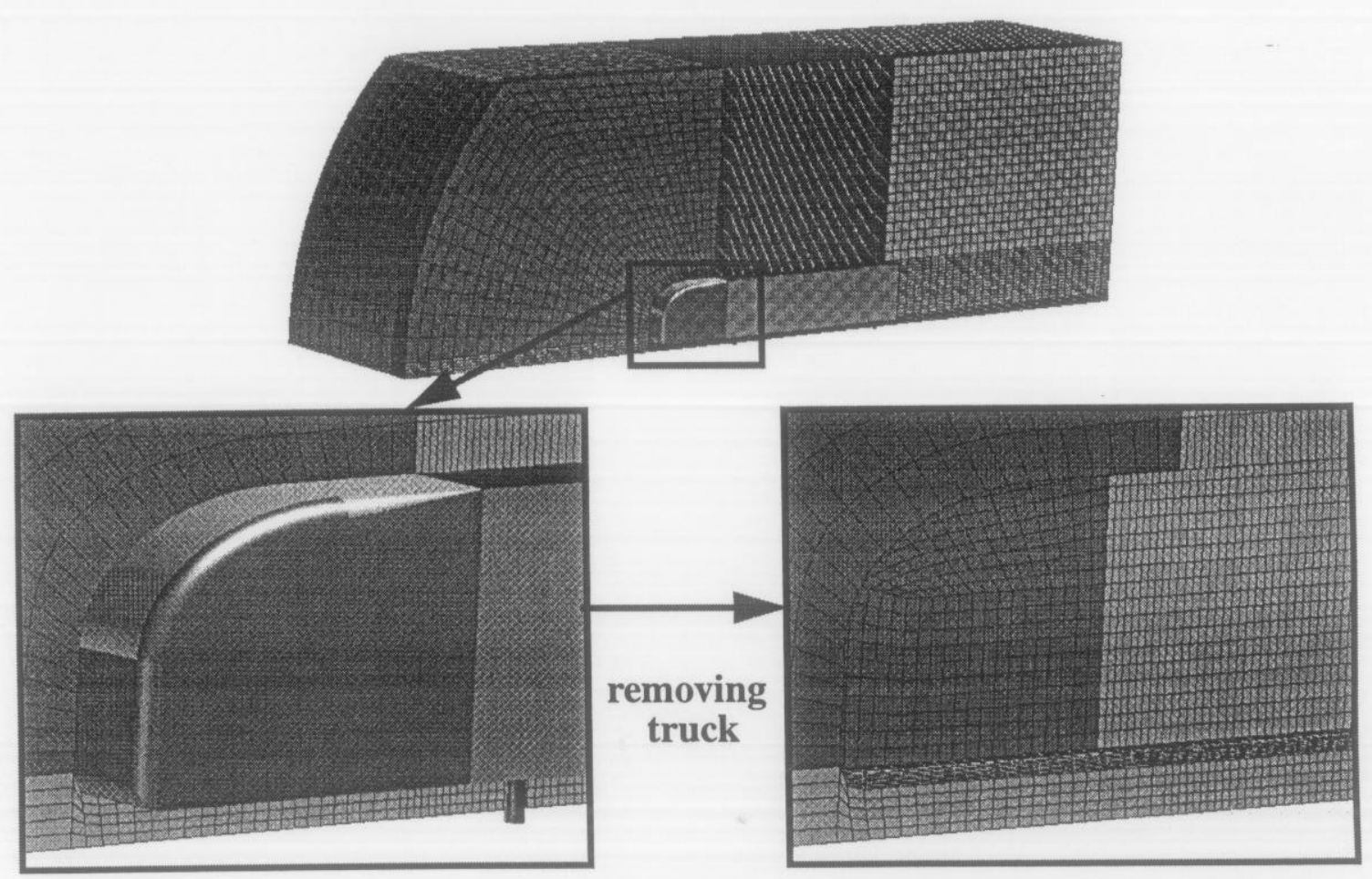

FIGURE 13. Example LES/FEM calculations demonstrate the benefits of the unstructured grid option and the ability of LES to capture the unsteady wake flow. 